\title{
Discovery of serum biomarkers for pancreatic adenocarcinoma using proteomic analysis
}

\author{
A Xue ${ }^{1,6}$, CJ Scarlett ${ }^{1,6,7}$, L Chung ${ }^{2}$, G Butturini ${ }^{3}$, A Scarpa ${ }^{4}$, R Gandy', SR Wilson ${ }^{5}$, RC Baxter ${ }^{2}$ and RC Smith ${ }^{*, 1}$ \\ 'Department of Surgery, University of Sydney, Royal North Shore Hospital, St Leonards, New South Wales 2065, Australia; ${ }^{2}$ Kolling Institute of Medical \\ Research, University of Sydney, Royal North Shore Hospital, St Leonards, New South Wales 2065, Australia; ${ }^{3}$ Surgical and Anaesthesiological Department, \\ University of Verona, Piazzale La Scuro, Verona 1037 I 34, Italy; ${ }^{4}$ Pathology Department, University of Verona, Piazzale La Scuro, Verona I 037 I 34, Italy; \\ ${ }^{5}$ Mathematical Sciences Institute, Australian National University, Canberra, Australian Capital Territory 0200, Australia
}

\begin{abstract}
BACKGROUND AND AIMS: The serum/plasma proteome was explored for biomarkers to improve the diagnostic ability of CAI9-9 in pancreatic adenocarcinoma (PC).

METHODS: A Training Set of serum samples from 20 resectable and 18 stage IV PC patients, 54 disease controls (DCs) and 68 healthy volunteers $(\mathrm{HV}$ s) were analysed by surface-enhanced laser desorption and ionisation time-of-flight mass spectrometry (SELDI-TOF MS). The resulting protein panel was validated on 40 resectable PC, 2 I DC and 19 HV plasma samples (Validation-I Set) and further by ELISA on 33 resectable PC, 28 DC and 18 HV serum samples (Validation-2 Set). Diagnostic panels were derived using binary logistic regression incorporating internal cross-validation followed by receiver operating characteristic (ROC) analysis.

RESULTS: A seven-protein panel from the training set PC vS DC and from PC vs HV samples gave the ROC area under the curve (AUC) of 0.90 and 0.90 compared with 0.87 and 0.9 I for CA 19-9. The AUC was greater $(0.97$ and 0.99, P<0.05) when CAI9-9 was added to the panels and confirmed on the validation-I samples. A simplified panel of apolipoprotein C-I (ApoC-I), apolipoprotein A-II (ApoA-II) and CAI9-9 was tested on the validation-2 set by ELISA, in which the ROC AUC was greater than that of CA 19-9 alone for PC vs DC (0.90 vs 0.84) and for PC vs HV (0.96 vs 0.90).

CONCLUSIONS: A simplified diagnostic panel of CA19-9, ApoC-I and ApoA-II improves the diagnostic ability of CAI9-9 alone and may have clinical utility.

British Journal of Cancer (2010) I 03, 39I-400. doi: 10.1038/sj.bjc.6605764 www.bjcancer.com

Published online 29 June 2010

(c) 2010 Cancer Research UK
\end{abstract}

Keywords: pancreatic adenocarcinoma; proteomics; serum biomarkers; apolipoproteins

Diagnostic serum biomarkers for pancreatic cancer are unsatisfactory (Honda et al, 2005), although many have been investigated (Grote and Logsdon, 2007). CA19-9, the 'gold standard' (Goonetilleke and Siriwardena, 2007), has a $79 \%$ (range reported in the literature $70-90 \%)$ sensitivity and $82 \%(68-91 \%)$ specificity. Although grossly elevated CA19-9 predicts unresectable disease (Maithel et al, 2008) and prognosis for chemoradiotherapy (Berger et al, 2008), 10-15\% of patients cannot produce CA19-9 because of Lewis-negative genotype (Kawai et al, 2008). In addition, serum CA19-9 is elevated in other malignancies and benign disorders (Kim et al, 2004). Recently, the necessity for a multivariate serum marker has been proposed (Grote and Logsdon, 2007).

The low-molecular-weight proteome $(<10 \mathrm{kDa})$ is a rich source of new potential biomarkers, but these do not resolve easily with 2D gel electrophoresis (Issaq et al, 2002; Wang et al, 2003). Mass spectrometric technologies perform optimally in the lowmolecular-weight range and early reports using surface-enhanced laser desorption and ionisation time-of-flight mass spectrometry

\footnotetext{
*Correspondence: Professor RC Smith; E-mail: rsmith@med.usyd.edu.au

${ }^{6}$ These authors made equal contributions to this project.

${ }^{7}$ Current address: Garvan Institute of Medical Research, Darlinghurst, New South Wales 2010, Australia.

Received 30 March 2010; revised 2 June 20I0; accepted 9 June 2010; published online 29 June 2010
}

(SELDI-TOF MS) have been promising (Bhattacharyya et al, 2004; Koopmann et al, 2004; Honda et al, 2005). Five groups have studied a serum protein panel using SELDI in combination with CA19-9 and have shown that such a combination of proteins is superior to CA19-9 alone (Koopmann et al, 2004; Honda et al, 2005; Ehmann et al, 2007; Guo et al, 2009; Navaglia et al, 2009).

Despite the similar findings of the above SELDI-based papers, there is no validated biomarker or biomarker panel for pancreatic adenocarcinoma (PC). Consequently, this paper describes an international study using SELDI-TOF MS to identify biomarkers that are followed by the use of ELISA to validate these on a further set of samples in which the disease controls (DCs) had severe pancreatic pathology. This work therefore aims to develop improved biomarkers that would be useful in the diagnosis of patients at an increased risk of pancreatic cancer.

\section{MATERIALS AND METHODS}

\section{Clinicopathological details and sample collection}

A total of 319 samples were obtained with patient consent. Training serum samples were obtained from 160 patients (the Training Set) managed at Centre-1 (Sydney, Australia) and their protein panels were validated against 80 plasma samples collected 
Table I Detail of patient groups

\begin{tabular}{|c|c|c|c|}
\hline & $\begin{array}{c}\text { Training } \\
\text { SELDI } \\
n\end{array}$ & $\begin{array}{c}\text { Validation-I } \\
\text { SELDI } \\
n\end{array}$ & $\begin{array}{c}\text { Validation-2 } \\
\text { ELISA } \\
n\end{array}$ \\
\hline Pancreatic ductal adenocarcinoma & 38 & 40 & 33 \\
\hline Male & 20 & 23 & 15 \\
\hline Female & 18 & 17 & 18 \\
\hline Stage 1 & 7 & - & । \\
\hline Stage lla & 5 & 4 & 7 \\
\hline Stage Ilb & 8 & 36 & 14 \\
\hline Stage IV & 18 & - & $\mid 1$ \\
\hline Disease control & 54 & 21 & 28 \\
\hline Male & 28 & 18 & 16 \\
\hline Female & 26 & 3 & 12 \\
\hline Cholelithiasis & 17 & 2 & \\
\hline Choledocholithiasis & & & 6 \\
\hline Intraductal papillary mucinous & 6 & - & I \\
\hline neoplasm & & & \\
\hline Carotid artery stenosis & - & 6 & \\
\hline Mucinous cystadenoma & 5 & - & 2 \\
\hline Neuroendocrine (islet cell) & 5 & - & $7(4)$ \\
\hline \multicolumn{4}{|l|}{ tumour } \\
\hline Gallstone pancreatitis & 4 & - & 2 \\
\hline Chronic pancreatitis & 4 & | & 2 \\
\hline Benign stricture & & & 2 \\
\hline Hernia & 3 & 2 & \\
\hline Acute cholecystitis & - & 3 & \\
\hline Other ${ }^{\mathrm{a}}$ & 10 & 7 & 3 \\
\hline Healthy volunteer & 68 & 19 & 18 \\
\hline Male & 28 & 12 & 9 \\
\hline Female & 40 & 7 & 9 \\
\hline
\end{tabular}

Abbreviation: SELDI = surface-enhanced laser desorption and ionisation. The number in brackets indicates malignant islet cell tumours. ${ }^{a}$ Other includes: Training Set - villous adenoma $(n=2)$, serous cystadenoma $(n=2)$, pancreatic pseudocyst $(n=2)$, solid pseudopapillary tumour $(n=1)$, Caroli's disease $(n=1)$, gastrooesophageal reflux $(n=1)$ and ruptured appendix $(n=1)$; Validation-I Set - leg ischaemia $(n=2)$, rectal bleeding $(n=2)$, diaphragmatic hernia $(n=1)$, phlebitis $(n=1)$ and haematological disorder $(n=1)$; and Validation-2 Set - solitary fibrous tumour, granulomata, intrapancreatic pseudocyst.

from subjects treated at Centre-2 (Verona, Italy; Validation-1 Set; Table 1). The samples were treated similarly with aliquots stored at $-80^{\circ} \mathrm{C}$ until analysis. Further confirmation of the findings was undertaken using ELISA methodology on a second set of 79 samples (Validation-2 Set), obtained from centre-1 from 33 PC patients, 28 DCs and 18 healthy volunteers (HVs). These controls were matched for age and sex. All DCs had pancreaticobiliary pathology requiring surgical or endoscopic intervention (Table 1).

Union Internationale Contre le Cancer (UICC) (Sobin, 2009) classification was used to stage the PC patients.

The study was approved by the ethics committees of the Northern Sydney Health Human Research, Sydney, Australia and the University of Verona, Verona, Italy.

\section{Preparation of serum or plasma for SELDI analysis}

Serum or plasma was diluted $1: 1$ with denaturing buffer $(8 \mathrm{M}$ urea/ $1 \%$ CHAPS), and then centrifuged at 12000 r.p.m. for $5 \mathrm{~min}$ ). The supernatant was diluted $1: 25$ with trifluoroacetic acid (TFA), added to $50 \%$ acetonitrile/ $0.5 \%$ TFA, spotted on a hydrophobic (H50) protein chip array and processed as previously described (Scarlett et al, 2006). The protein chip arrays were analysed using the Bio-Rad Protein Biological System IIc ProteinChip Reader (Bio-Rad Hercules, CA, USA).

\section{SELDI-TOF MS analysis}

Mass spectra were generated in the $m / z$ range $2500-75000$ with a laser intensity setting of 220 (arbitrary units) and detector sensitivity set at 8 . The laser was optimised for $4000-20000 \mathrm{~m} / \mathrm{z}$ peaks, whereas peaks $<1000 \mathrm{~m} / z$ were deflected from the detector. Mean values from duplicate samples were used in subsequent analyses. The $\mathrm{m} / \mathrm{z}$ value for each of the peaks was determined using external calibration with known standards (Sigma-Aldrich, St Louis, MO, USA): bovine insulin $(5734.51+1 \mathrm{H})$, equine cytochrome $c(12361.96+1 \mathrm{H})$, equine apomyoglobin $(16952.27+1 \mathrm{H})$ and rabbit muscle aldolase $(39212.28+1 \mathrm{H})$. Spectra were analysed using the Ciphergen Protein Chip Software Version 3.1 (Bio-Rad).

\section{Protein purification and identification}

The proteins of interest were size fractionated on a Superose $12 \mathrm{HR}$ 10/300 GL column (Amersham Pharmacia Biotech, Uppsala, Sweden), equilibrated and eluted with $0.1 \mathrm{M}$ acetic acid/ $0.1 \mathrm{M} \mathrm{NaCl}$ $(\mathrm{pH} 3.0)$ and fractions were monitored on SELDI using normal phase NP20 chips. Pooled fractions containing maximum activity were subjected to reverse-phase high-performance liquid chromatography (HPLC) on a $4.6 \times 250 \mathrm{~mm}$ Jupiter $5 \mu \mathrm{m}, 300 \AA$ C1 8 column (Phenomenex, Torrance, CA, USA) after $30 \mathrm{~min}$ of gradient elution $(15-60 \%$ acetonitrile in $0.1 \%$ TFA), and fractions were again monitored by SELDI-TOF MS on NP20 chips. The fraction containing the peaks of interest was lyophilised and then sent to the Bioanalytical Mass Spectrometry Facility (University of New South Wales, Sydney, Australia) for identification by tryptic peptide mass fingerprinting and MS sequencing. To confirm the protein identities, a SELDI immunoadsorption approach was performed. In this instance, a rabbit polyclonal Apolipoprotein C-I (ApoC-I) antibody (AbCam, Cambridge, UK) was bound to an RS100 protein chip array and analysed on the SELDI-TOF MS.

The three protein peaks at $m / z 16989,17132$ and 17247 were identified by a different strategy from above, following the report of Ehmann et al (2007), which described protein peaks at $\mathrm{m} / \mathrm{z}$ 17270 and 17390 in human serum SELDI profiles as apolipoprotein A-II (ApoA-II) homodimers. The serum of patients suffering from PC and the purified human plasma ApoA-II (Sigma-Aldrich, Paris, France) were directly submitted to an RS100 preactivated chip coupled with ApoA-II antibody (AbCam, Paris, France).

\section{Western blotting validation}

PC serum samples and purified human plasma ApoA-II were treated with $20 \mu \mathrm{M}$ dithiothreitol (DTT) from 0 to $4 \mathrm{~h}$, respectively, and subjected to western blotting analysis with anti-ApoA-II antibody (AbCam) as described in a previous study (Xue et al, 2009).

\section{Statistical analysis}

The raw peak intensity data were normalised using the total ion current between 2500 and $75000 \mathrm{~m} / z$ and peak detection was performed using the Biomarker Wizard utility (Version 3.1, Bio-Rad). Sample group statistics were performed on peak intensity values for profiles of PCs vs DCs and HVs. Univariate analysis of individual peaks was performed using the nonparametric Mann-Whitney $U$-test with significance considered at $P<0.05$. The discriminatory power for each marker was characterised by receiver operating characteristic (ROC) area under the curve (AUC) analysis and the AUCs were compared using the Hanley and McNeil method (Hanley and McNeil, 1983; SPSS software Version 12.0, Chigaco, IL, USA). Values of ROC AUC are presented (Scarlett et al, 2006) with their 95\% confidence intervals.

Co-correlation of protein peaks was examined by Spearman's non-parametric correlation coefficients because of the wide 
variance of the data for protein values, CA19-9 and bilirubin values.

\section{Development and validation of candidate biomarker models}

Development Biomarker panels were developed on the centre-1 serum cohort $(n=160)$ using the multivariate binary logistic regression with ten-fold cross-validation technique previously described (Scarlett et al, 2006), which had been developed by Ambroise and McLachlan (Ambroise and McLachlan, 2002). Only serum peaks that significantly discriminated PCs from DCs or from HVs at $P<0.01$ were considered for multivariate analysis. This repeated random sampling procedure allowed for the correction of selection bias and enabled the calculation of unbiased estimates of sensitivity and specificity, overall accuracy and ROC AUC values with their $95 \%$ confidence intervals of the candidate biomarker panels.

Validation The models developed in the training phase were then tested on the independent centre-2 plasma samples $(n=80)$. Likelihood ratios (LRs) were calculated for each model to estimate the ratio of the likelihood of the test result in patients with disease to the likelihood of the same test result in patients without disease. Results with an LR of $>10$ or $<0.1$ effect a substantial change on disease likelihood over a broad range of pre-test estimates, whereas an LR of 1.0 leaves the likelihood of disease unchanged (Brown and Reeves, 2003).

\section{ELISA measurement of serum levels of CA19-9, ApoA-II and ApoC-I}

Having developed the protein biomarker panel with SELDI, further confirmation was sought using ELISA as follows. Duplicate serum or plasma levels of CA19-9 were measured by ELISA kit (Alpha Diagnostic International, San Antonio, TX, USA) for which normal values were set at $<37 \mathrm{U} \mathrm{ml}^{-1}$. Similarly, levels of ApoA-II and ApoC-I were measured by AssayMax ApoA-II ELISA kit and AssayMax ApoC-I ELISA kit (AssayPro, St Charles, MO, USA), respectively. Absorbance was measured at $450 \mathrm{~nm}$ on a microplate reader (Tecan, Salzburg, Austria) within $10 \mathrm{~min}$.

\section{RESULTS}

\section{Patient characteristics}

Three separate groups of subjects were studied. The initial training set from centre- 1 consisted of 160 samples: 38 from PC patients (21 studied before surgery and 17 before palliative treatments), 54 DC samples from patients with other pancreatico-biliary disorders and 68 samples from HVs (Table 1). Although 18 of 39 PC and 10 of 54 DC patients had elevated serum bilirubin values, there was a significant difference between the PC and DC groups for mean bilirubin and other liver function test values (Table 2). The validation-1 samples from centre-2 were obtained from $40 \mathrm{PC}$ subjects taken before resection, $21 \mathrm{DCs}$ from patients requiring an acute admission and $21 \mathrm{HV}$ subjects (Table 1). Of the PC patients from centre-2, 20 had elevated serum bilirubin values.

Validation-2 samples were from a further 79 patients from centre-1: 33 PC samples (21 from patients who underwent resection), 28 DC subjects (18 underwent pancreatic resection whereas 10 had therapeutic endoscopic retrograde cholangiopancreatography (ERCP)) and $18 \mathrm{HV}$ subjects. The DCs and HVs had similar age and sex distribution (Table 1). In the validation-2 groups, bilirubin was elevated in 21 of 33 with PCs and 10 of 28 DCs $\left(\chi^{2}=2.1\right.$, NS), but the median values were marginally different $(P=0.047)$. The HVs had normal liver function values.

\section{Serum CA19-9 values of training and validation-1 samples}

Blood CA19-9 levels were elevated in 63 of 78 PCs, 20 of 75 DCs and 15 of 85 HVs with greater mean ( \pm s.e.m.) values in PC compared with DC and HV patients $($ CA19-9 = $1192.2( \pm 477.0)$, 34.3 ( \pm 5.0$)$ and $19.7 \mathrm{U} \mathrm{ml}^{-1}( \pm 2.1)$, respectively; $\left.P<0.001\right)$. Mean CA19-9 values were also higher in the stage IIb and IV patients $\left(2212 \pm 1354 \mathrm{U} \mathrm{ml}^{-1}\right)$ compared with the early stage I and IIa patients $\left(163 \pm 19 \mathrm{U} \mathrm{ml}^{-1}\right)$, but this was not statistically significant using the Mann-Whitney test.

\section{Development of diagnostic models from SELDI analysis}

Differences in SELDI profiles were observed between PC, DC and HV subjects using the $\mathrm{H} 50$ protein chip array (Supplementary Figure S1). In all, 62 individual protein peaks observed in the $\mathrm{m} / \mathrm{z}$ range 3000-20000 were analysed. In the training set samples, 21

Table 2 Mean and s.e.m. of age and biochemical indices for patient groups

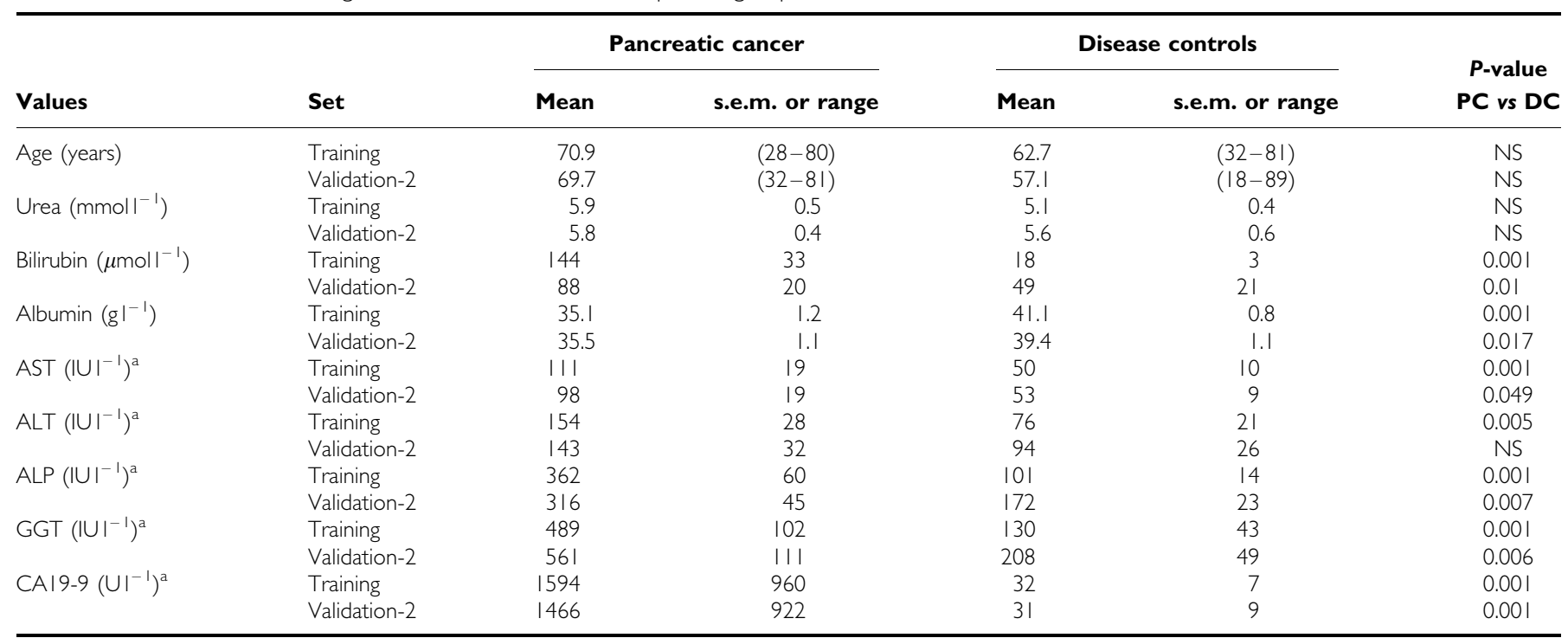

Abbreviations: ALP = alkaline phosphatase; $A S T=$ glutamic-oxaloacetic transaminase; $A L T=$ glutamic-pyruvic transaminase; $C A \mid$ 9-9 = carbohydrate antibody 19-9; $\mathrm{DC}=$ disease control; GGT $=\gamma$-glutamyltransferase; $\mathrm{NS}=$ not significant; $\mathrm{PC}=$ pancreatic cancer. ${ }^{\mathrm{a}}$ Results are for serum values. $P$-value indicates $\mathrm{Mann}-\mathrm{Whitney} \mathrm{U}$-test result. 
peaks were found to be differentially expressed between PCs and DCs. These individual putative tumour markers had ROC AUC values ranging from 0.62 to 0.81 , with 14 upregulated in $\mathrm{PC}$ patients (Supplementary Table S1). Logistic regression with tenfold cross-validation was applied to the subset of protein peaks that were differentially expressed between PCs and DCs to a significance of $P<0.01$ as determined by univariate analysis (Supplementary Table S1). This selected a panel of seven protein peaks $(m / z 6420,8451,8614,9137,9626,9694$ and 12 862) that correctly classified $74 \%$ of PC and $87 \%$ of DC serum samples (ROC AUC: 0.90 (0.85-0.96), Supplementary Table S1 and Figure 1A). Discriminatory power was further improved by including the values of the tumour marker CA19-9 into the model. This model correctly classified $89 \%$ of PC and $96 \%$ of DC serum samples (ROC AUC: 0.97 (0.93-0.99), Supplementary Table S1 and Figure 1A).

When the protein peak model was applied to the validation-1 samples, $90 \%$ of PC and $67 \%$ of DC samples were correctly classified (ROC AUC: 0.88 (0.79-0.96), Supplementary Table S1 and Figure 1B). When CA19-9 was included in the model, $93 \%$ of PC and $71 \%$ of DC samples were correctly classified (ROC AUC: $0.93(0.86-0.99))$. For both the training and validation-1 cohorts, the ROC AUC values were significantly increased by the addition of CA19-9 $(P<0.05)$ and were significantly greater than that for CA19-9 alone (Figures 2C and D).

\section{PC vs HV serum}

In all, 18 peaks were found to be differentially expressed between PCs and HVs, 13 with a $P$-value $\leqslant 0.01$, using logistic regression analysis (Supplementary Table S1). The ten-fold cross-validation approach selected a final panel of four peaks $(\mathrm{m} / z$ 6618, 16989, 17132 and 17247 ) that correctly classified $71 \%$ of PC and $96 \%$ of HV serum samples (ROC AUC: 0.90 (0.84-0.96), Supplementary Table S1 and Figure 1C), which was similar to the AUC of CA19-9 (ROC AUC: $0.914(0.839-0.989))$. When CA19-9 was added to the protein panel, the discriminatory power was better $(P<0.05)$ than for the protein panel or CA-19-9 alone - 92\% of PC and $97 \%$ of $\mathrm{HV}$ samples were correctly classified (ROC AUC: 0.99 $(0.98-1.00))$.

When the logistic regression equation from the training set was applied to the validation- 1 sample set, $90 \%$ of PC and $74 \%$ of HV serum samples were correctly classified (ROC AUC: 0.90 (0.81-0.98), Supplementary Table S1 and Figure 1D). Again, the addition of CA19-9 significantly improved $(P<0.05)$ the classification, identifying $93 \%$ of $\mathrm{PC}$ and $84 \%$ of $\mathrm{HV}$ samples (ROC AUC: $0.96(0.91-1.00)$ ), which was greater than that for CA19-9 alone (ROC AUC: $0.81(0.61-0.92)$, but this did not reach significance.

\section{Testing for co-correlation of the protein values}

Spearman's correlation coefficients for the variables selected in the above protein panels (Supplementary Table S2) indicate that these proteins were associated with one of two groups by close correlation. One group was closely correlated with the $\mathrm{m} / z 6420$ peak and the others were closely correlated with the $\mathrm{m} / z 17247$ peak. Subsequent analysis of the identities of these peaks clarified this correlation.
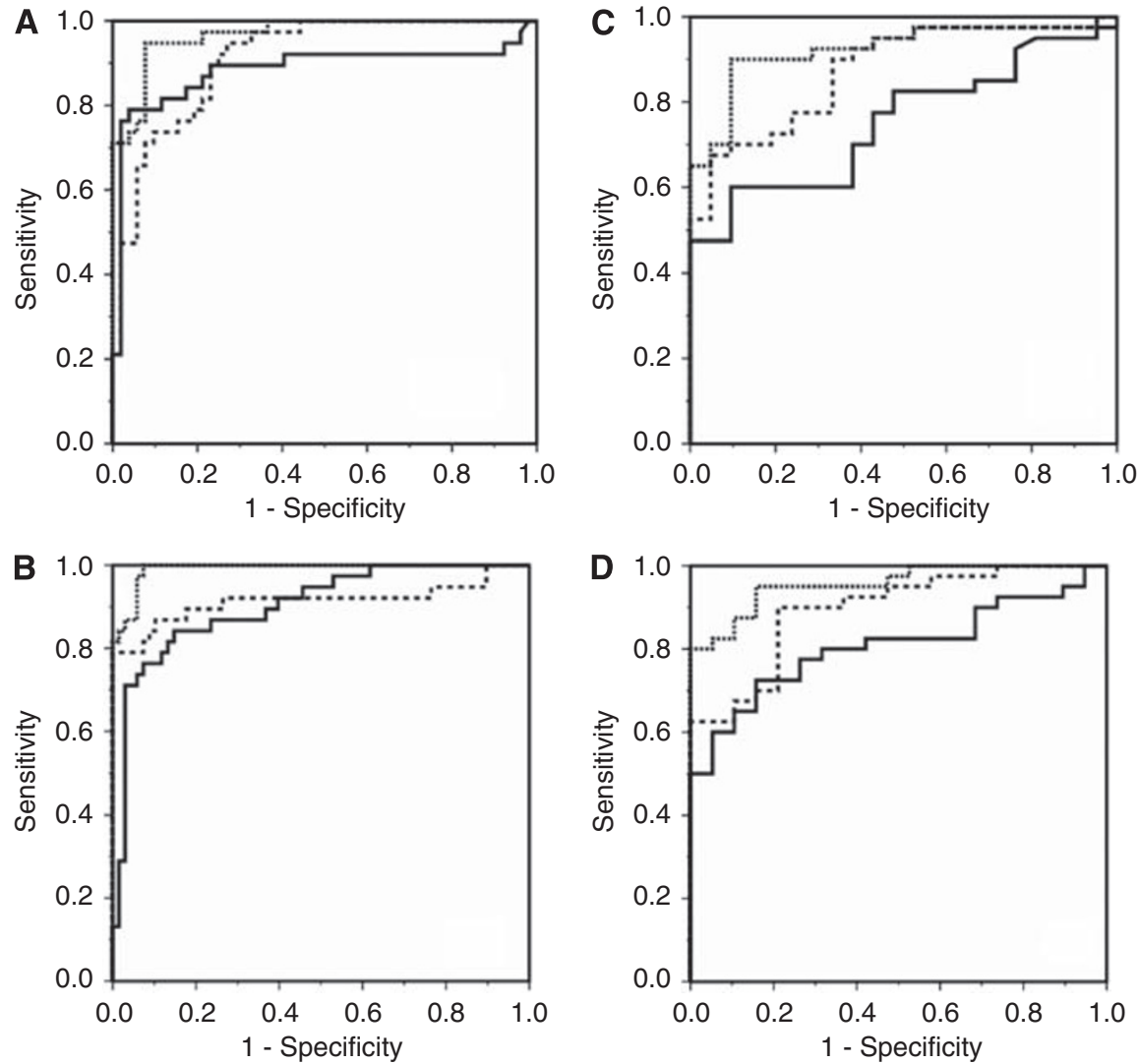

Figure I Receiver operator characteristic (ROC) curves for discrimination of pancreatic adenocarcinoma (PC) from disease controls (DCs) and healthy volunteers (HVs) in both the centre-I and centre-2 cohorts. The protein panel derived from ten-fold cross-validation (solid line), CA 19-9 (dashed line) and biomarker panel + CAI9-9 (dotted line). (A) PC vs DC (centre-I): ROC AUC $=0.90$ for the biomarker panel, 0.87 for CAI9-9 and 0.97 for the biomarker panel + CA19-9. (B) PC vs DC (centre-2): ROC AUC =0.88 for the biomarker panel, 0.75 for CAI9-9 and 0.93 for the biomarker panel + CA 19-9. (C) PC vs HV (centre-I): ROC AUC =0.90 for the biomarker panel, 0.9I for CAI9-9 and 0.99 for the biomarker panel + CA I9-9. (D) PC vs HV (centre-2): ROC AUC $=0.90$ for the biomarker panel, $0.8 \mathrm{I}$ for CAI9-9 and 0.96 for the biomarker panel + CAI9-9. 
A

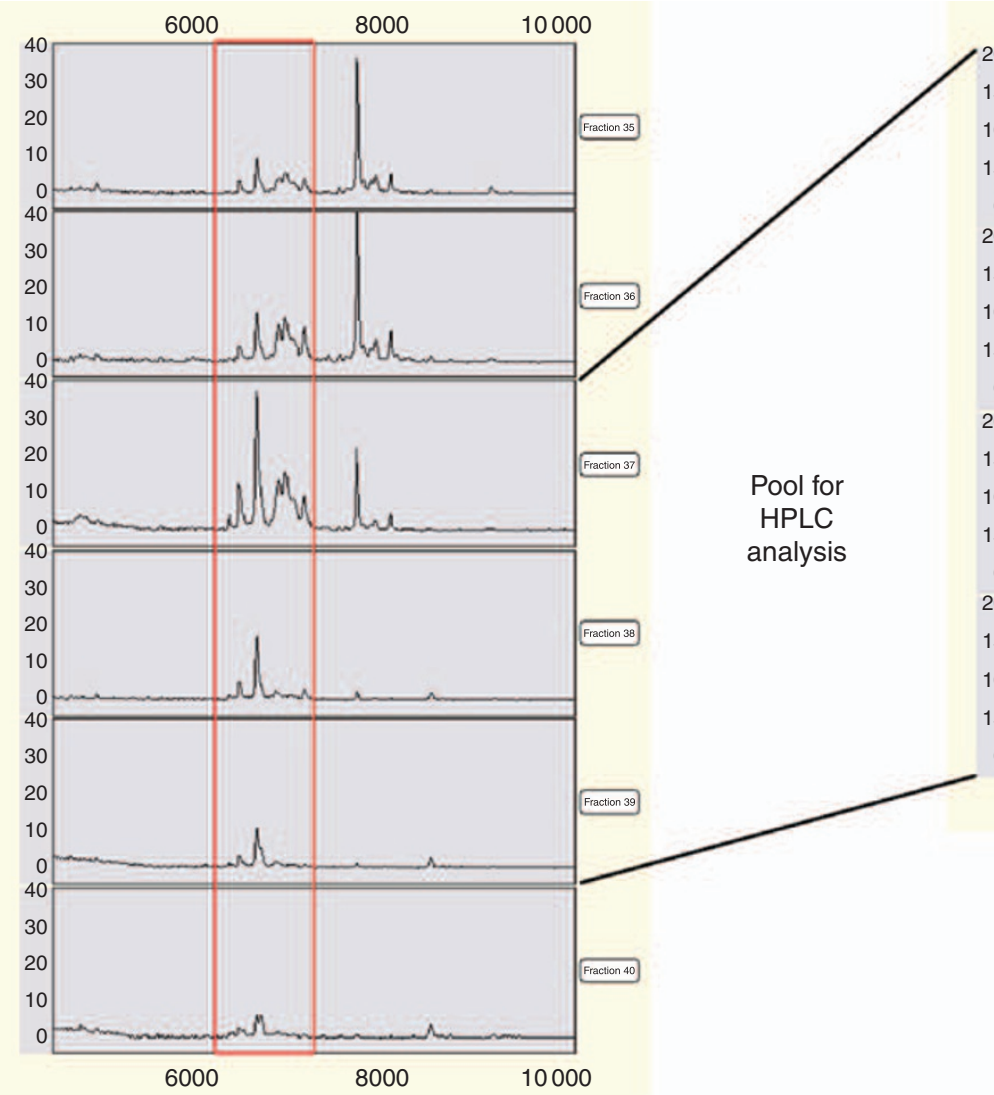

B 


\section{Protein purification and identification}

Serum samples that showed high $\mathrm{m} / \mathrm{z} 6420$ and 6618 peak intensities were purified for identification of the apparent $6.6 \mathrm{kDa}$ proteins. The protein fractions were monitored on SELDI using NP20 chips (Figure 2A). Pooled fractions containing maximum activity were further purified using HPLC and again monitored on NP20 chips (Figure 2B). The fractions containing the peaks of interest were lyophilised for identification by tryptic peptide mass fingerprinting and MS sequencing. The $\mathrm{m} / \mathrm{z} 6618$ peak was identified as ApoC-I from three peptides that covered $38.5 \%$ of the amino acid sequence. To confirm the protein identities obtained from sequencing, a SELDI immunoadsorption approach was performed using an ApoC-I antibody bound to an RS100 protein chip array. The $\mathrm{m} / z 6420$ and 6618 peaks were captured with high intensity with minimal nonspecific binding observed for the affinity-purified IgG control (Figure 2C).

The $m / z$ 8614, 16989,17185 and 17247 proteins were confirmed as ApoA-II isoforms by examining samples of serum and purified human ApoA-II. Human ApoA-II not only showed the appearance of three major isoforms, $m / z 16989,17185$ and 17247 , but also of a peak at 8614. A similar pattern was observed in the plasma specimens (Figure $3 \mathrm{Ae}$ ) in SELDI MS profile. Using SDS-PAGE according to Gillard et al (2005), specimens of purified ApoA-II and serum samples from PC patients were reduced for $3 \mathrm{~h}$ using $20 \mu \mathrm{m}$ DTT. This resulted in a loss of the $17-\mathrm{kDa}$ band and an increase in the $8.6-\mathrm{kDa}$ band, which is consistent with reduction of disulfide-linked homodimers.

The PC serum samples and purified human plasma ApoA-II were treated with $20 \mu \mathrm{M}$ DTT from 0 to $4 \mathrm{~h}$. The reduced samples were then submitted to western blotting analysis with anti-ApoA-II antibody. Figure 3B shows that both crude serum and purified ApoA-II contained the ApoA-II dimer $(\sim 17.2 \mathrm{kDa})$ and the bands disappeared after reduction by DTT. Furthermore, the band at $8.6 \mathrm{kDa}$ was concomitantly increased in intensity upon DTT treatment. Figures $3 \mathrm{C}$ and $\mathrm{D}$ show the differences in concentration of ApoC-I and ApoA-II in non-reducing conditions according to Gillard et al (2005) on western blot and the semiquantitative expression undertaken in 15 serum samples from each of the PC, DC and HV subjects.

\section{Simplified diagnostic panel}

Because of the high correlation coefficients between all of the proteins in the diagnostic panels to either ApoC-I or ApoA-II, a simplified diagnostic panel was proposed combining the ApoC-I $\mathrm{m} / z 6420$ peak and the ApoA-II $\mathrm{m} / z 8614$ with CA19-9. This combination was used to examine the diagnostic effectiveness in the four different patient groups and showed strong ROC AUC values of 0.99 for PC $v s \mathrm{HV}$ in the training set, 0.95 for PC $v s \mathrm{HV}$ in the validation- 1 set, 0.94 for $\mathrm{PC} v s \mathrm{DC}$ in the training set and 0.92 for PC vs DC subjects in the validation-1 set (Figures 4A-D). The positive and negative LRs for the above comparisons were 16.5, 5.4, 6.7 and 4.7 and $0.06,0.11,0.07$ and 0.19 , respectively, indicating that this test would make a useful contribution to clinical decision making but would not be useful for screening purposes.

\section{ELISA validation}

ELISA mean (s.e.m.) measurements on the HV, DC and PC validation-2 set for ApoA-II were 55 (2), 47 (1) and $44 \mathrm{gl}^{-1}$ (1) and
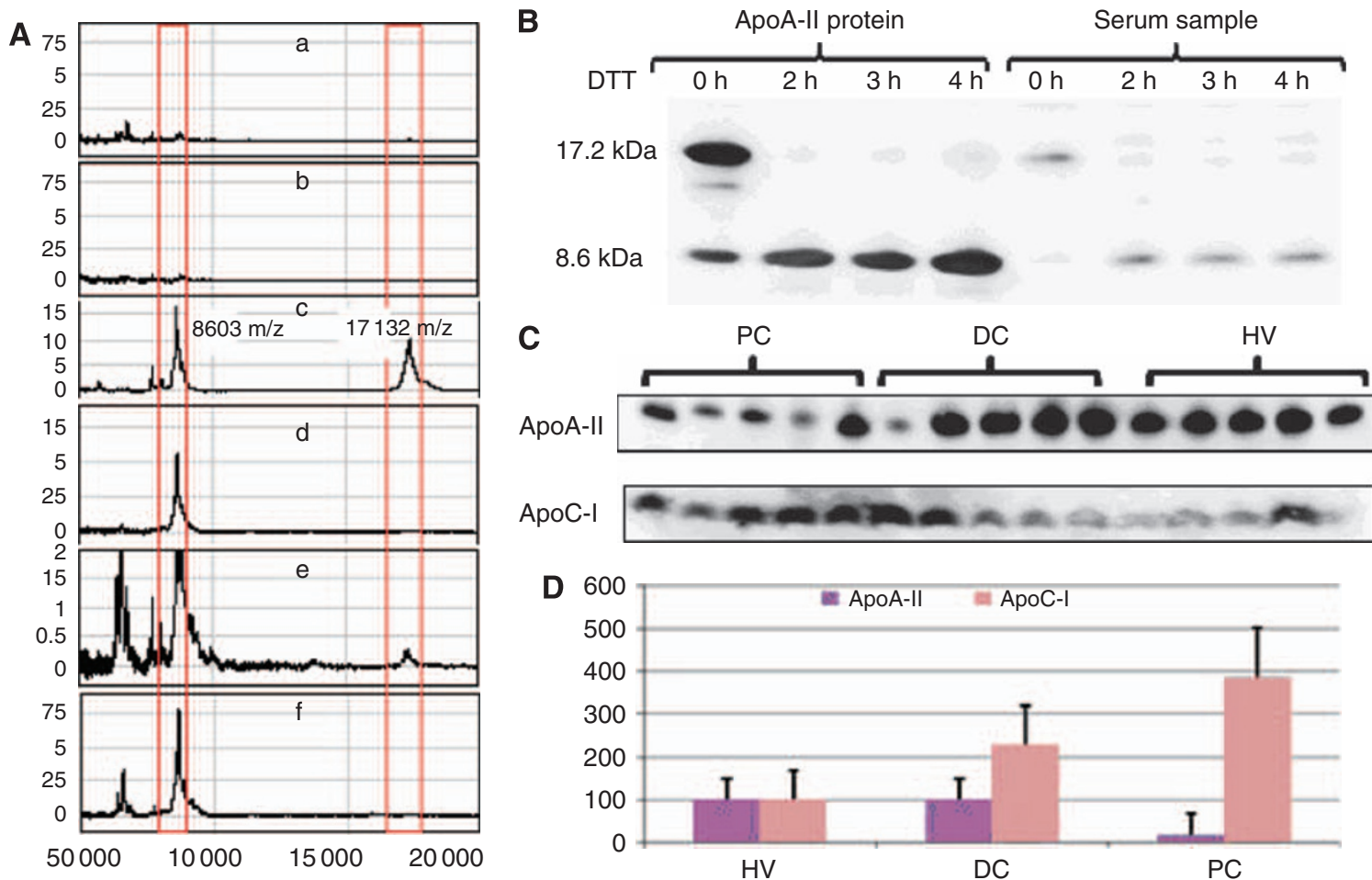

Figure 3 (A) Plots of peak height against $\mathrm{m} / \mathrm{z}$ for the identification of ApoA-II isoforms using SELDI RSI00 profiles. (a) ApoA-II purified human plasma ApoA-II (Sigma-Aldrich) IgG, (b) serum without IgG, (c) purified human ApoA-II on ApoA-II antibody-enriched chip indicating a number if peaks 8603 indicating monomer and 17 I 32 indicating dimer, (d) purified ApoA-II treated with $20 \mu \mathrm{M}$ DTT for $3 \mathrm{~h}$ on antibody-enriched chip, (e) serum on ApoA-II antibody-enriched chip and ( $f$ ) purified ApoA-Il treated with $20 \mu \mathrm{M}$ DTT for $3 \mathrm{~h}$ on antibody-enriched chip. (B) Western blotting analysis of the effect of 0 to $4 \mathrm{~h}$ of $20 \mu \mathrm{M}$ DTT reduction on a serum sample and on purified human plasma ApoA-II. (C) Westem blot of the expression of ApoC-I and $\sim 17 \mathrm{kDa}$ ApoA-II in five pancreatic cancer samples (PC), five disease control samples (DC) and five human volunteer samples (HV). (D) Plot of mean and s.d. band intensity (\% of HV) as semiquantified by Pro-Gel scanning (Gel-Pro Analyzer Properties) for PC samples $(n=15)$, DC samples $(n=15)$ and HV samples $(n=15)$. 

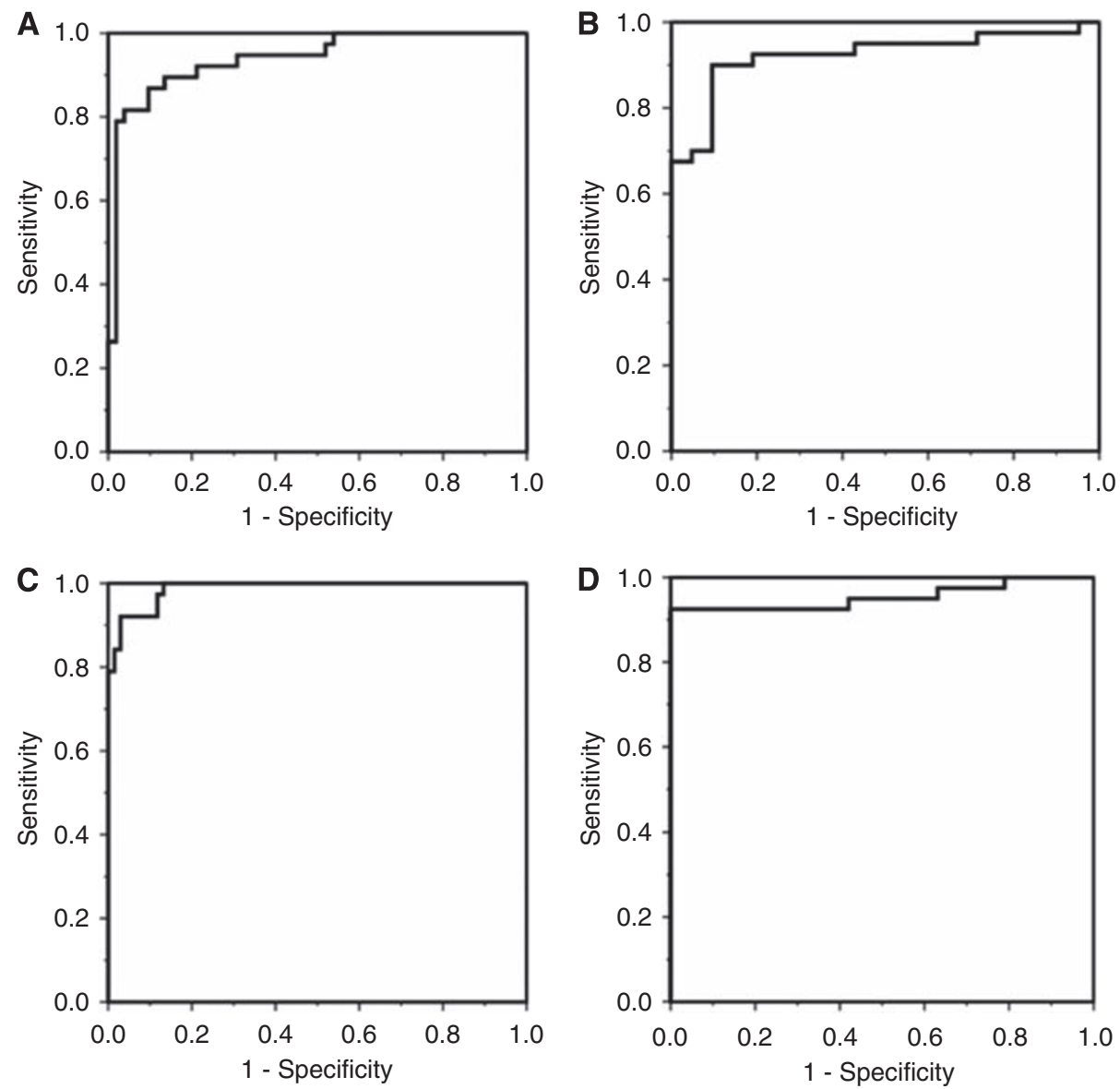

Figure 4 ROC curves for the performance of the simplified biomarker panel (CAI9-9 and the protein peak heights for $\mathrm{m} / \mathrm{z} 6420$ (ApoC-I) and I7247 proteins (ApoA-II)). (A-D) PC vs DC for centre-I samples, PC vs DC for centre-2 samples, PC vs HV for centre-I samples and PC vs HV for centre-2 samples are shown (see text for AUC values).
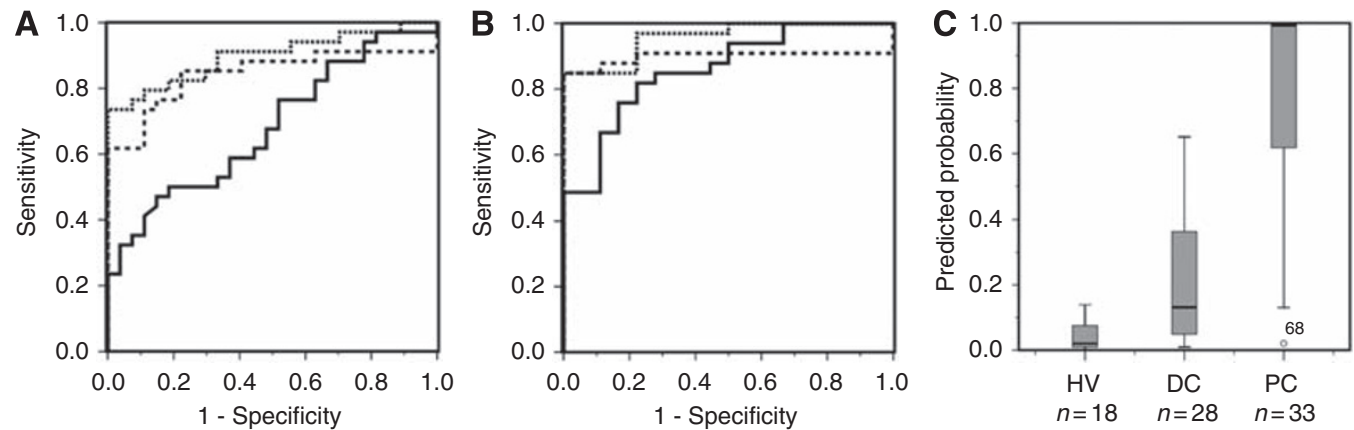

Figure 5 ROC curves for the ELISA measures on the validation-2 set. (A) PC vs HV and (B) PC vs DC. Dotted lines represent the curves for combined ApoA-II, ApoC-I and CA 19-9, dashed lines indicate CAI9-9 whereas solid lines indicate the combined protein panel of ApoA-II and ApoC-I (see text for AUC values). (C) The probability of diagnosing PC as separate from benign cases (combined HV and DC) using binary logistic regression on the ELISA values of ApoA-II, ApoC-I and CA19-9.

for ApoC-I were 107 (4), 101 (6) and $124 \mathrm{gl}^{-1}$ (12). For ApoC-1 the difference between DCs and PCs indicated a trend to significance at $P=0.07$ and the mean ApoA-II values were different between HVs and PCs $(P=0.001)$, whereas the mean differences between DCs and PCs were not significant. Although there were significant differences between the PC and DC patients in the values of bilirubin and other liver function tests, these were less significant than those from the training sets (Table 2). ROC analysis confirmed a significant diagnostic influence of the combination of two proteins, ApoA-II and ApoC-I, with AUC of 0.86 $(0.76-0.96)$ for $\mathrm{PC} v s \mathrm{HV}$ and $0.68(0.55-0.81)$ for $\mathrm{PC} v s \mathrm{DC}$ patients. The addition of these proteins to CA19-9 improved the ROC AUC compared with CA19-9 alone to $0.96(0.90-1.0)$ vs 0.90 $(0.80-0.99)$ for $\mathrm{HV}$ samples and $0.90(0.82-0.98)$ vs 0.84 (0.74-0.95) for DC samples (Figure 5). Multivariate stepwise backward analysis showed significance for the combined panel but no influence from the addition of bilirubin, albumin or serum alkaline phosphatase was observed. Figure 5C shows the probability of diagnosing pancreatic cancer compared with benign disease (HV and DC cases combined), derived from binary logistic regression using the ELISA values for ApoA-II, ApoC-I and CA19-9. 


\section{DISCUSSION}

This international collaborative study shows the utility of SELDITOF MS for identification of potential diagnostic biomarker panels that have been confirmed on two independent sample sets. The significant proteins in the diagnostic panel were identified as ApoA-II and ApoC-I. This allowed for a simplified panel, the efficacy of which was confirmed on validation-2 samples by ELISA. Although this study has similar motives to previous studies using SELDI-TOF MS (Koopmann et al, 2004; Honda et al, 2005; Ehmann et al, 2007; Guo et al, 2009; Navaglia et al, 2009), important new findings are described. Both ApoC-I (Takano et al, 2008) and ApoA-II (Ehmann et al, 2007) have been identified in previous studies, but they have not been used in combination, in which they seem to provide independent contributions to the diagnostic panel. The sensitivities and specificities of new individual peaks were similar to that of CA19-9 in discriminating PCs from both DCs and HVs, but importantly discriminatory power was significantly improved by multi-protein marker models that included CA-19-9. The robustness of the model was shown by the fact that although developed on a training set of serum samples, it was able to be validated on plasma samples (Banks et al, 2005). Further support for the diagnostic capacity of this protein panel was confirmed by ELISA on a separate sample set.

Both the PC $v s$ DC and PC vs HV classification models improved the discriminatory power when compared with CA19-9 alone. The importance of CA19-9 as a diagnostic marker of pancreatic cancer (Goonetilleke and Siriwardena, 2007) is confirmed by this study, but its discriminating ability is significantly improved by the SELDI-derived diagnostic panels. Furthermore, the overall accuracy of the combined results indicates that these diagnostic models may become clinically useful in high-risk patient groups. However, because of the low prevalence of pancreatic cancer, a much greater accuracy would be required before these diagnostic panels could be clinically useful in an asymptomatic population (Bhattacharyya et al, 2004).

ApoA-II was observed in both the monomeric form of $m / z 8614$ and in the dimers of $m / z 16989,17185$ and 17247 in the SELDI MS profiles. In human plasma or serum, ApoA-II mainly exists as a disulfide-linked homodimer at approximately $17.3 \mathrm{kDa}$ (Gillard et al, 2005). There seems to be a number of isoforms that may account for the smaller adjacent peaks of $m / z 16989$ and 17132 . The apolipoproteins lie on the surface of lipid particles and have an important role in directing the fate of these particles to different organs for metabolism. The precise function of ApoA-II is not entirely clear but it is suggested that ApoA-II is rich in HDL particles that promote the formation of atheroma (Stein et al, 1995). The exact metabolism of ApoA-II is not completely known. It has a diagnostic role in prostate carcinoma that has been shown to release ApoA-II (Malik et al, 2005). In comparison with pancreatic cancer, in which low levels are diagnostic, in prostate cancer high levels are diagnostic.

Five research groups using SELDI-TOF for the identification of serum markers of pancreatic cancer have also developed multiple marker panels that include CA19-9 (Koopmann et al, 2004; Honda et al, 2005; Ehmann et al, 2007; Guo et al, 2009; Navaglia et al, 2009). Koopmann et al (2004) used IMAC-Cu ${ }^{2+}$ and weak cationic exchange protein chips and found a two-peak panel that differentiated PC samples from HVs, whereas a three-peak panel distinguished PCs from DCs. These panels performed better than CA19-9 alone, but when used in combination, the diagnostic accuracy was further improved. Guo et al, (2009) used strong anion-exchange protein chips to show that the three protein biomarkers, $m / z 4155,4791$ and 28068 , are more accurate than CA19-9 in differentially diagnosing pancreatic cancer. The larger peak was identified as the protein C14orf16 that is highly expressed in cancer tissue, although the function of this protein is not fully elucidated. Yu et al (2005) used the Biomarker Patterns software
(Bio-Rad) to develop a classification tree that includes six peaks that differentiate cancer from non-cancer with high sensitivity and specificity without CA19-9 in 100 subjects, using IMAC-Cu ${ }^{2+}$ protein chips, and recently this group has shown high sensitivity and specificity of the protein panel. Honda et al (2005) used three protein chip types (IMAC-Cu ${ }^{2+}, \mathrm{H} 50$ and CM10) on 24 subjects, and developed training models using a support vector machine algorithm that selected four peaks, three from the CM10 protein chip and one from the H50, which when combined could distinguish pancreatic cancer from healthy controls with high sensitivity and specificity. The SELDI-derived panel was superior to that of CA19-9 alone, but was further improved in combination with CA19-9. A fifth recent study compared serum from cancer patients and healthy volunteers and showed that a panel of three proteins had a sensitivity of $83 \%$ and a specificity of $96 \%$ when tested in an independent validation set of samples. Interestingly, ApoA-II was identified, along with ApoA-I and transthyretin (Ehmann et al, 2007). These proteins are produced by the liver and have important roles in lipid transport, and although their full function is not understood, they are components of HDL. Although these studies report differing numbers and peaks that constitute the diagnostic panels that show promise, each lacks the identification of many of the proteins of interest.

Our findings that lipoproteins are perturbed in pancreatic caner suggest that lipid metabolism is important for such tumours. This is supported by cell culture studies that show greater cell growth in the presence of lipid (Wang et al, 2009). Therefore, it is interesting that the apolipoproteins seen to be markers of pancreatic cancer are both involved with lipid transport. ApoA-II is a component of HDL subclass 3 in which it combines with ApoA-I and results in a smaller particle size by increasing its hydrophobic properties (Gao et al, 2009). This acts as a counter to the cardio-protective effects of ApoA-I. However, the exact function of ApoA-II is unclear (Blanco-Vaca et al, 2001; Gao et al, 2009). It is possible that ApoA-II helps direct lipid to the cancer. However, there are a number of alternative reasons that the concentration of apolipoproteins may be depressed in patients with pancreatic cancer: weight loss (Ng et al, 2010), liver dysfunction (Tacikowski et al, 2000) and diabetes (Onat et al, 2009). It will be important to study this further to determine whether this effect is directly related to PC demand for nutrition or whether they are an epiphenomenon of the weight loss associated with the cancer.

This study found that the $m / z 6618$ peak, identified as ApoC-I, was most frequently selected for the PC $v s$ HV model by ten-fold cross-validation. Investigation of the serum/plasma MS literature revealed that ApoC-I exists in human serum as ApoC-I $(\sim 6630 \mathrm{Da})$ and a truncated isoform that lacks N-terminal Thr-Pro- (6432 Da) (Jin and Manabe, 2005). ApoC-I has an important role in controlling plasma lipid metabolism, but little is known about its role in the cancer process (Bondarenko et al, 1999). Genetic upregulation of ApoC-I has been shown in gastric cancer (Oue et al, 2004), whereas in a recent breast cancer study ApoC-I formed part of a multi-protein index (developed from SELDI analysis) that could predict metastatic relapse in high-risk primary breast cancer patients receiving adjuvant chemotherapy (Goncalves et al, 2006). ApoC-I has also been shown in pancreatic cancer and is suggested to be associated with infiltrating macrophages within the juxtatumoural stroma (Ricci et al, 2005). Ricci et al (2005) suggested that this may be an indication of direct communication between stroma and cancer cells and provides evidence of a response to infiltrative growth that may predominate in tumour-stromal interactions independent of cancer type.

The inflammatory process associated with cancer may have important prognostic implications (Pine et al, 2009) and the specificity of this may provide opportunities for biomarker discovery. Using electrospray ionisation (ESI) ion-trap tandem MS (ESI-MS) to explore the differences between human pancreatic 
cancer sera and normal sera, Hanas et al (2008) revealed greater heterogeneity in cancer sera, especially in the low-mass region. Using a statistical bootstrap approach, they showed that three large-mass proteins involved in inflammatory responses were elevated in pancreatic cancer sera: $\alpha-2$ macroglobulin, ceruloplasmin and complement 3C. Firpo et al (2009) also improved the discriminatory power of CA19-9 when used with two acute-phase proteins: haptoglobin and serum amyloid A. In our validation-2 data, ApoC-I levels correlated with white cell count; although ApoC-I is an inflammatory marker, the results confirm those of Takano et al (2008) that ApoC-I is also a potentially useful marker for pancreatic cancer.

This international collaborative study confirms the usefulness of SELDI-TOF MS for exploration of low-mass proteome (Guo et al, 2009) in which there are important signatures of the cancer process (Navaglia et al, 2009) when PC is resectable. Because of the complex cancer process, a panel of biomarkers is likely to be useful in high-risk groups of patients. Further exploration of the exact function of these proteins may provide insights into PC. It will be important to discover how early in the tumourigenic process these proteins become altered, and whether these panels of proteins are capable of diagnosing curable PC.

\section{ACKNOWLEDGEMENTS}

Dr Anthony Gill from the Department of Anatomical Histopathology at Royal North Shore Hospital provided expert pathological detail on the surgical specimens from that hospital. Dr Sally Smith provided help with the paper. This study was supported by the Cure Cancer Australia Foundation and the Cancer Surgery Research Foundation (CanSur). CJS is the recipient of a University of Sydney Postgraduate Award and is a Cancer Institute NSW Fellowship. The University of Verona Surgical Department was supported through the Associazione Italiana per la Ricerca sul Cancro (AIRC), Italy; Fondazione Cassa di Risparmio di Verona (Bando 2004); Ministeri Università-Ricerca e Salute, Rome, Italy; European Community Grant PL018771; and Fondazione Giorgio Zanotto, Verona, Italy.

\section{REFERENCES}

Ambroise C, McLachlan GJ (2002) Selection bias in gene extraction on the basis of microarray gene-expression data. Proc Natl Acad Sci USA 99(10): 6562

Banks RE, Stanley AJ, Cairns DA, Barrett JH, Clarke P, Thompson D, Selby PJ (2005) Influences of blood sample processing on low-molecularweight proteome identified by surface-enhanced laser desorption/ ionization mass spectrometry. Clin Chem 51(9): 1637

Berger AC, Garcia Jr M, Hoffman JP, Regine WF, Abrams RA, Safran H, Konski A, Benson III AB, MacDonald J, Willett CG (2008) Postresection CA 19-9 predicts overall survival in patients with pancreatic cancer treated with adjuvant chemoradiation: a prospective validation by RTOG 9704. J Clin Oncol 26(36): 5918

Bhattacharyya S, Siegel ER, Petersen GM, Chari ST, Suva LJ, Haun RS (2004) Diagnosis of pancreatic cancer using serum proteomic profiling. Neoplasia 6(5): 674-686

Blanco-Vaca F, Escola-Gil JC, Martin-Campos JM, Julve J (2001) Role of apoA-II in lipid metabolism and atherosclerosis: advances in the study of an enigmatic protein. J Lipid Res 42(11): 1727-1739

Bondarenko PV, Cockrill SL, Watkins LK, Cruzado ID, Macfarlane RD (1999) Mass spectral study of polymorphism of the apolipoproteins of very low density lipoprotein. J Lipid Res 40(3): 543-555

Brown MD, Reeves MJ (2003) Evidence-based emergency medicine/skills for evidence-based emergency care. Interval likelihood ratios: another advantage for the evidence-based diagnostician. Ann Emerg Med 42(2): 292

Ehmann M, Felix K, Hartmann D, Schnolzer M, Nees M, Vorderwulbecke S, Bogumil R, Buchler MW, Friess H (2007) Identification of potential

\section{AUTHOR CONTRIBUTIONS}

A Xue was involved in study concept and design, acquisition of data, analysis and interpretation of data, drafting of the paper, critical revision of the paper for important intellectual content and statistical analysis.

CJ Scarlett was involved in study concept and design, acquisition of data, analysis and interpretation of data, drafting of the paper, critical revision of the paper for important intellectual content, statistical analysis and also obtained funding.

L Chung was involved in technical and material support, study supervision and also approved the paper.

$G$ Butturini was involved in acquisition and interpretation of data, and critical revision of the paper for important intellectual content, and also obtained funding and material support.

A Scarpa was involved in critical revision of the paper for important intellectual content, and obtained funding and material support.

$\mathrm{R}$ Gandy was involved in technical and material support, and approved the paper.

SR Wilson was involved in study concept and design, analysis and interpretation of data, critical revision of the paper for important intellectual content, statistical analysis and study supervision.

RC Baxter was involved in study concept and design, analysis and interpretation of data, critical revision of the paper for important intellectual content, study supervision, and also obtained funding.

RC Smith was involved in study concept and design, acquisition of data, analysis and interpretation of data, drafting of the paper, critical revision of the paper for important intellectual content, statistical analysis, study supervision, and also obtained funding, technical or material support.

\section{Conflict of interest}

The authors declare no conflict of interest.

Supplementary Information accompanies the paper on British Journal of Cancer website (http://www.nature.com/bjc)

markers for the detection of pancreatic cancer through comparative serum protein expression profiling. Pancreas 34(2): 205-214

Firpo MA, Gay DZ, Granger SR, Scaife CL, DiSario JA, Boucher KM, Mulvihill SJ (2009) Improved diagnosis of pancreatic adenocarcinoma using haptoglobin and serum amyloid A in a panel screen. World J Surg 33(4): 716

Gao X, Yuan S, Jayaraman S, Gursky O (2009) Differential stability of high-density lipoprotein subclasses: effects of particle size and protein composition. J Mol Biol 387(3): 628

Gillard BK, Chen YS, Gaubatz JW, Massey JB, Pownall HJ (2005) Plasma factors required for human apolipoprotein A-II dimerization. Biochemistry 44(2): 471

Goncalves A, Esterni B, Bertucci F, Sauvan R, Chabannon C, Cubizolles M, Bardou VJ, Houvenaegel G, Jacquemier J, Granjeaud S, Meng XY, Fung ET, Birnbaum D, Maraninchi D, Viens P, Borg JP (2006) Postoperative serum proteomic profiles may predict metastatic relapse in high-risk primary breast cancer patients receiving adjuvant chemotherapy. Oncogene 25(7): 981

Goonetilleke KS, Siriwardena AK (2007) Systematic review of carbohydrate antigen (CA 19-9) as a biochemical marker in the diagnosis of pancreatic cancer. Eur J Surg Oncol 33(3): 266-270

Grote T, Logsdon CD (2007) Progress on molecular markers of pancreatic cancer. Curr Opin Gastroenterol 23(5): 508

Guo J, Wang W, Liao P, Lou W, Ji Y, Zhang C, Wu J, Zhang S (2009) Identification of serum biomarkers for pancreatic adenocarcinoma by proteomic analysis. Cancer Sci 100(12): 2292 
Hanas JS, Hocker JR, Cheung JY, Larabee JL, Lerner MR, Lightfoot SA, Morgan DL, Denson KD, Prejeant KC, Gusev Y, Smith BJ, Hanas RJ, Postier RG, Brackett DJ (2008) Biomarker identification in human pancreatic cancer sera. Pancreas 36(1): 61

Hanley JA, McNeil BJ (1983) A method of comparing the areas under receiver operating characteristic curves derived from the same cases. Radiology 148(3): 839-843

Honda K, Hayashida Y, Umaki T, Okusaka T, Kosuge T, Kikuchi S, Endo M, Tsuchida A, Aoki T, Itoi T, Moriyasu F, Hirohashi S, Yamada T (2005) Possible detection of pancreatic cancer by plasma protein profiling. Cancer Res 65(22): 10613-10622

Issaq HJ, Conrads TP, Janini GM, Veenstra TD (2002) Methods for fractionation, separation and profiling of proteins and peptides. Electrophoresis 23(17): $3048-3061$

Jin Y, Manabe T (2005) Direct targeting of human plasma for matrixassisted laser desorption/ionization and analysis of plasma proteins by time of flight-mass spectrometry. Electrophoresis 26(14): 2823

Kawai S, Suzuki K, Nishio K, Ishida Y, Okada R, Goto Y, Naito M, Wakai K, Ito Y, Hamajima N (2008) Smoking and serum CA 19-9 levels according to Lewis and secretor genotypes. Int J Cancer 123(12): 2880

Kim JE, Lee KT, Lee JK, Paik SW, Rhee JC, Choi KW (2004) Clinical usefulness of carbohydrate antigen 19-9 as a screening test for pancreatic cancer in an asymptomatic population. J Gastroenterol Hepatol 19(2): 182-186

Koopmann J, Zhang Z, White N, Rosenzweig J, Fedarko N, Jagannath S, Canto MI, Yeo CJ, Chan DW, Goggins M (2004) Serum diagnosis of pancreatic adenocarcinoma using surface-enhanced laser desorption and ionization mass spectrometry. Clin Cancer Res 10(3): 860-868

Maithel SK, Maloney S, Winston C, Gonen M, D'Angelica MI, Dematteo RP, Jarnagin WR, Brennan MF, Allen PJ (2008) Preoperative CA 19-9 and the yield of staging laparoscopy in patients with radiographically resectable pancreatic adenocarcinoma. Ann Surg Oncol 15(12): 3512

Malik G, Ward MD, Gupta SK, Trosset MW, Grizzle WE, Adam BL, Diaz JI, Semmes OJ (2005) Serum levels of an isoform of apolipoprotein A-II as a potential marker for prostate cancer. Clin Cancer Res 11(3): 1073

Navaglia F, Fogar P, Basso D, Greco E, Padoan A, Tonidandel L, Fadi E, Zambon CF, Bozzato D, Moz S, Seraglia R, Pedrazzoli S, Plebani M (2009) Pancreatic cancer biomarkers discovery by surface-enhanced laser desorption and ionization time-of-flight mass spectrometry. Clin Chem Lab Med 47(6): 713

Ng TW, Chan DC, Barrett PH, Watts GF (2010) Effect of weight loss on HDL-apoA-II kinetics in the metabolic syndrome. Clin Sci (Lond) 118(1): 79

Onat A, Hergenc G, Ayhan E, Ugur M, Can G (2009) Impaired antiinflammatory function of apolipoprotein A-II concentrations predicts metabolic syndrome and diabetes at 4 years follow-up in elderly Turks. Clin Chem Lab Med 47(11): 1389
Oue N, Hamai Y, Mitani Y, Matsumura S, Oshimo Y, Aung PP, Kuraoka K, Nakayama H, Yasui W (2004) Gene expression profile of gastric carcinoma: identification of genes and tags potentially involved in invasion, metastasis, and carcinogenesis by serial analysis of gene expression. Cancer Res 64(7): 2397-2405

Pine JK, Fusai KG, Young R, Sharma D, Davidson BR, Menon KV, Rahman SH (2009) Serum C-reactive protein concentration and the prognosis of ductal adenocarcinoma of the head of pancreas. Eur J Surg Oncol 35(6): 605

Ricci F, Kern SE, Hruban RH, Iacobuzio-Donahue CA (2005) Stromal responses to carcinomas of the pancreas: juxtatumoral gene expression conforms to the infiltrating pattern and not the biologic subtype. Cancer Biol Ther 4(3): 302

Scarlett CJ, Smith RC, Saxby A, Nielsen A, Samra JS, Wilson SR, Baxter RC (2006) Proteomic classification of pancreatic adenocarcinoma tissue using protein chip technology. Gastroenterology 130(6): 1670-1678

Sobin L (2009) TNM Classification of Malignant Tumours. Wiley-Blackwell: Hoboken, NJ

Stein O, Dabach Y, Hollander G, Ben-Naim M, Oette K, Stein Y (1995) Effects of interactions of apolipoprotein A-II with apolipoproteins A-I or A-IV on $[3 \mathrm{H}]$ cholesterol efflux and uptake in cell culture. Biochim Biophys Acta 1257(2): 174

Tacikowski T, Milewski B, Dzieniszewski J, Nowicka G, Walewska-Zielecka B (2000) Comparative analysis of plasma lipoprotein components assessed by ultracentrifugation in primary biliary cirrhosis and chronic hepatitis. Med Sci Monit 6(2): 325

Takano S, Yoshitomi H, Togawa A, Sogawa K, Shida T, Kimura F, Shimizu H, Tomonaga T, Nomura F, Miyazaki M (2008) Apolipoprotein C-1 maintains cell survival by preventing from apoptosis in pancreatic cancer cells. Oncogene 27(20): 2810

Wang F, Kumagai-Braesch M, Herrington MK, Larsson J, Permert J (2009) Increased lipid metabolism and cell turnover of MiaPaCa2 cells induced by high-fat diet in an orthotopic system. Metabolism 58(8): 1131

Wang YY, Cheng P, Chan DW (2003) A simple affinity spin tube filter method for removing high-abundant common proteins or enriching low-abundant biomarkers for serum proteomic analysis. Proteomics 3(3): $243-248$

Xue A, Xue M, Jackson C, Smith RC (2009) Suppression of urokinase plasminogen activator receptor inhibits proliferation and migration of pancreatic adenocarcinoma cells via regulation of ERK/p38 signaling. Int J Biochem Cell Biol 41(8-9): 1731

Yu Y, Chen S, Wang LS, Chen WL, Guo WJ, Yan H, Zhang WH, Peng CH, Zhang SD, Li HW, Chen GQ (2005) Prediction of pancreatic cancer by serum biomarkers using surface-enhanced laser desorption/ionizationbased decision tree classification. Oncology 68(1): 79-86 